\title{
Modelagem dos Efeitos Geodinâmicos que afetam as Medições Maregráficas e GNSS
}

Modeling of geodynamic effects affecting tide and GNSS measurements

\author{
Fabio Luiz Albarici 1 \\ Gabriel Nascimento Guimarães ${ }^{2}$ \\ Jorge Luiz Alves Trabanco ${ }^{3}$ \\ Marcelo Santos 4
}

Recebido em agosto de 2018.

Aprovado em janeiro de 2019.

\begin{abstract}
RESUMO
Os estudos dos diferentes tipos de marés têm se tornado mais importantes por sua aplicação na determinação precisa da órbita de satélites artificiais e na manutenção das coordenadas precisas de estações Global Navigation Satellite System (GNSS). Esta pesquisa fornece a modelagem de algumas componentes geodinâmicas que influenciam diretamente na solidez das estações GNSS e maregráficas, além de inserir erros na determinação precisa do Nível Médio do Mar (NMM). Utilizando-se da abordagem do Posicionamento por Ponto Preciso (PPP) para verificação do deslocamento da crosta e da modelagem da maré terrestre e carga oceânica, o objetivo deste trabalho é analisar e quantificar cada uma das forças geodinâmicas que atuam no marégrafo e na estação GNSS RBMC-NEIA. Os modelos analisados para a carga oceânica e maré terrestre, bem como o deslocamento da crosta, condizem com a influência geodinâmica aplicada à estação e ao marégrafo, onde verifica-se que os mesmos possuem pouca movimentação vertical e o NMM mantém baixa variação, aumentando de forma gradual ao longo do tempo.
\end{abstract}

PALAVRAS-CHAVE: Maré Terrestre. Maré Oceânica. Nível Médio do Mar.

\footnotetext{
ABSTRACT

Studies of the different types of tides have become more important because of their application in the precise determination of the orbit of artificial
}

\footnotetext{
${ }^{1}$ IFSULDEMINAS - Campus Inconfidentes. Programa de Pós-graduação em Engenharia Civil/FECUNICAMP, Brasil. E-mail: fabio.albarici@ifsuldeminas.edu.br

${ }^{2}$ Universidade Federal de Uberlândia - Campus Monte Carmelo, Programa de Pós-Graduação em Agricultura e Informações Geoespaciais. Brasil. E-mail: gabriel@ufu.br

${ }^{3}$ Faculdade de Engenharia Civil/UNICAMP, Brasil. E-mail: trabanco@fec.unicamp.br

${ }^{4}$ University of New Brunswick. Postgraduate Program in Department of Geodesy and Geomatics Engineering, Canada. E-mail: msantos@unb.ca
} 
satellites and in the maintenance of the precise coordinates of Global Navigation Satellite System (GNSS) stations. This research provides the modeling of some geodynamic components that directly influence the solidity of the GNSS and tide stations, as well as inserting errors in the accurate determination of the Mean Sea Level (MSL). Using the Precision Point Positioning (PPP) approach to verify the crustal displacement and the modeling of the earth Tide and ocean tide load, the objective of this work is to analyze and quantify each of the geodynamic forces that act in the tide gauge and the GNSS station RBMC-NEIA. The models analyzed for the ocean tide load and the earth tide, as well as the crust displacement, are in agreement with the geodynamic influence applied to the station and the tide gauge, where it is verified that they have little vertical movement and the MSL maintains a low variation, increasing gradually over time.

KEYWORDS: Earth Tide. Ocean Tide. Mean Sea Level.

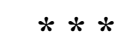

\section{Introdução}

O deslocamento de partículas líquidas (maré oceânica) e as deformações visco-elásticas da crosta terrestre (maré terrestre), sob a ação gravitacional da Lua e do Sol, constituem um assunto de muita relevância por suas implicações na Oceanografia, Geodésia e, mais recentemente, na Engenharia (GEMAEL, 2002).

Segundo Agnew (2007), carga de maré é a deformação da crosta terrestre causada pela redistribuição da massa oceânica sobre ela, ou seja, as águas oceânicas em seu movimento periódico exercem uma pressão ou carga sobre a crosta terrestre provocando deslocamentos verticais. As variações de carga oceânica são consequência da força gravitacional exercida principalmente pelo Sol e pela Lua nos oceanos. A Lua é o que tem maior efeito, principalmente nos períodos de Lua Cheia e Nova, quando as marés atingem o seu máximo. $\mathrm{O}$ efeito da carga oceânica no posicionamento de uma estação provoca um deslocamento de $10 \mathrm{~cm}$ na vertical e $3 \mathrm{~cm}$ na horizontal (próximo à costa). Estes valores podem variar de acordo com a posição geográfica da estação. Em regiões com até $1000 \mathrm{~km}$ da costa o deslocamento 
pode alcançar $1 \mathrm{~cm}$, e para distâncias maiores o efeito da carga oceânica pode ser desconsiderado (RAMOS et al., 2013).

A Terra, a Lua e o Sol participam de um processo gravitacional interativo do qual resultam esforços diferenciais significativos desde o interior até as porções mais superficiais do planeta. As variações de distância do Sol e da Lua em relação à Terra devido à movimentação dos astros, produzem uma alteração na gravidade. Este fenômeno é conhecido como força de maré e a ação desta força em cada porção elementar do planeta causa deformações com características visco-elásticas, variáveis no espaço e no tempo (BRETREGER e MATHER, 1978; CORDINI, 1998).

Por definição, maré oceânica é o fenômeno da subida e da descida do nível das águas de uma região devido aos efeitos gravitacionais criados pelos corpos celestes. A Lei da Gravitação Universal afirma que dois corpos materiais se atraem com forças cuja intensidade é diretamente proporcional ao produto de suas massas e inversamente proporcional ao quadrado da distância que os separa, conforme a Equação 1:

$$
F=\frac{G M m}{d^{2}}
$$

onde $G$ é a constante gravitacional, $M$ e $m$ são as massas dos dois corpos materiais e $d$ é a distância que os separam.

O efeito de maré ocorre num planeta porque a gravidade é função da distância radial, sendo que as diferentes partes do planeta estão a distâncias diferentes de um corpo perturbador, sofrendo forças gravitacionais ligeiramente distintas. Isso implica que partes diferentes do planeta se movem a acelerações também diferentes. $\mathrm{O}$ resultado disso é que o planeta é deformado de acordo com a atração gravitacional e é acelerado na direção que liga os dois centros (LAZZARO, 1989).

Essa força gravitacional faz com que os astros orbitem, além de produzir deformações em sua forma. Apesar do nome parecer paradoxal, 
ocorrem, de fato, marés terrestres, ou seja, a crosta terrestre se deforma como resultado desta força, do mesmo modo que ocorre com os oceanos, porém, em menor escala (BAKER et al., 1996). O método observacional que levou à demonstração da existência de marés terrestres foi baseado no raciocínio: marés oceânicas são observadas em relação a pontos fixos na crosta com a ajuda de marégrafos. Estes pontos seriam efetivamente fixos se o globo fosse perfeitamente rígido, o que faria com que a amplitude da maré oceânica observada fosse igual à calculada teoricamente. Ao contrário, se a parte sólida também se deforma, a amplitude medida será igual à diferença entre a maré oceânica e a maré terrestre, dessa forma descobriu-se que a amplitude da maré terrestre é três vezes menor do que a dos oceanos. (MELCHIOR, 1983).

As marés terrestres podem ser modeladas com uma precisão de poucos milímetros devido às observações realizadas na Terra, utilizando-se de equipamentos como os gravímetros (HOOIJBERG, 2008). A medida das marés terrestres é realizada indiretamente através da observação da gravidade na superfície terrestre, da extensão da deformação da crosta e das inclinações da vertical local, dessa forma a descrição das deformações é desenvolvida em termos dos carregamentos e respostas superficiais, as quais podem partir da formulação das deformações elásticas (CORDINI, 1998).

Os números de Love são parâmetros chave para se calcular as perturbações de maré terrestre. Esses números adimensionais foram introduzidos e aproximadamente estimados por Love no começo do século XX, e utiliza-se do fator gravimétrico (equação 2), onde permitem relacionar valores observados e valores teóricos (CORDINI, 1998).

$$
\delta=-\left(1+h-\frac{3}{2} k\right)
$$

Onde $k$ representa os efeitos de atração gravitacional do Sol e da Lua, o efeito da perturbação de maré na atração gravitacional da Terra; $h$ representa o efeito do deslocamento radial do gravímetro. 
Esse estudo tem se tornado importante para as mais diversas aplicações satelitais, incluindo a Geofísica, onde os mais variados satélites artificiais que orbitam a Terra requerem órbitas determinadas com muita precisão. Essas órbitas por sua vez sofrem influência das marés terrestres e oceânicas que causam perturbações adicionais no movimento dos satélites artificiais (PINTO, 2005). Além disso, o estudo de marés possui aplicações na engenharia (ex. obras portuárias), pois o aumento da precisão nas medidas requer correções dos efeitos de maré que antigamente eram ignorados (AGNEW, 2007).

Este aumento da precisão é verificado nas estações GNSS onde se observa um deslocamento vertical devido à deformação da crosta terrestre, causadas por marés oceânicas e terrestres. Este efeito pode atingir vários centímetros (SEEBER, 2003).

O potencial de maré gerado pela Lua e Sol pode ser descrito, pela Equação 3 (XU, 2007):

$$
W_{p}=u_{m}\left(\frac{1}{r^{\prime}}-\frac{1}{r}-\frac{\rho}{r^{2}} \cos z\right)
$$

Onde: $r$ é a distância geocêntrica da Lua, $\rho$ é a distância geocêntrica do ponto $p, u_{m}$ é a constante gravitacional da Lua, $z$ é o ângulo geocêntrico do zênite da Lua, e $r^{\prime}$ é a distância entre o ponto $p$ e a Lua.

Estas deformações na crosta produzida pela maré terrestre são caracterizadas pelas componentes horizontal (maré extensimétrica) e vertical (maré gravimétrica). É evidente que qualquer interpretação das observações de maré terrestre deve levar em consideração um conhecimento adequado dos efeitos das marés oceânicas (CORDINI, 1998).

Nesta pesquisa são utilizados, no processamento, as observações maregráficas, gravimétricas e GNSS. Assim, realizaram-se os cálculos através de softwares específicos (por exemplo: o PACMARÉ foi utilizado para obter-se os níveis médios diários, e Tsof e ETERNA34 para o cálculo da maré 
terrestre), e estimou-se como estas observações interagem entre si (por exemplo: carga de maré e maré terrestre), com o objetivo de analisar e quantificar cada uma das forças geodinâmicas que atuam no marégrafo e na estação GNSS de Cananeia/SP.

Nesta pesquisa, utilizou-se de uma série histórica de 5 anos (2012 a 2016) de observações GNSS e maregráficas, mais onze meses (março de 2013 a janeiro de 2014) de medições gravimétricas, para modelagem da maré terrestre e carga oceânica.

\section{Material e Métodos}

1.1 Área de estudo

O objeto de estudos é a estação maregráfica localizada na Base de Pesquisa "Dr. João de Paiva Carvalho", no município de Cananeia-SP, sob supervisão do Instituto Oceanográfico da USP.

$\mathrm{Na}$ estação maregráfica existe uma boa infraestrutura implantada (estação gravimétrica), e equipamentos instalados para observações da variação oceânica (marégrafo Kalesto) e possível movimentação da crosta terrestre (estação da Rede Brasileira de Monitoramento Continuo - NEIA (RBMC-NEIA)), que contribuíram para o objetivo desta pesquisa.

Cada um destes equipamentos fornece informações (dados) que foram utilizadas na determinação de valores para fins específicos (por exemplo, os dados da RBMC permitem obter a movimentação da crosta). A Figura 1 ilustra o local do estudo: 
Figura 1 - Área de estudo - Cananeia/SP

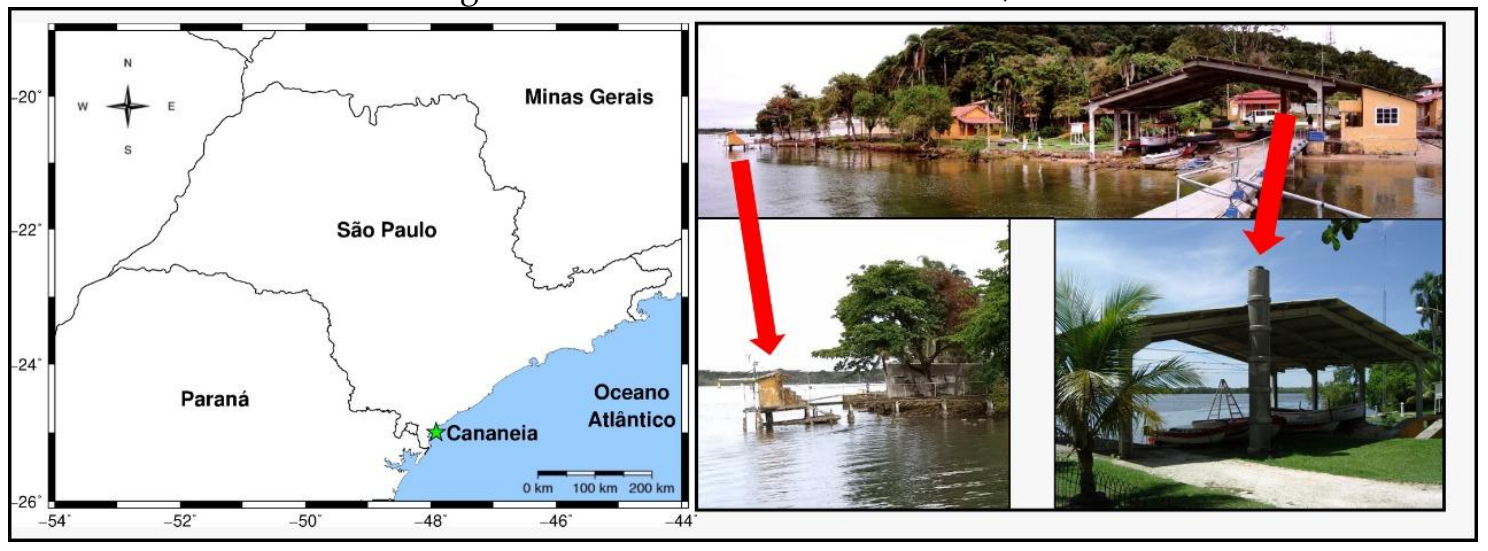

Fonte: elaborada pelos autores.

\subsection{Maré Terrestre}

$\mathrm{Na}$ coleta de dados de gravidade utilizou-se o gravímetro gPhone fabricado pela LaCoste \& Romberg, o qual utiliza um sistema de mola suspensa. Uma característica deste equipamento é sua baixa deriva instrumental, o que possibilita seu uso na integração de sinais periódicos, como os de maré terrestre e oceânicas por longos períodos, com precisão de 1 $\mu$ GAL (microgal). Os dados coletados por este gravímetro são gravados no sistema de aquisição como dados brutos, contendo anomalias. Essas anomalias são causadas por perturbações no ambiente produzindo interferências de sinais de alta frequência. Além disso, há descontinuidade de informações geradas por quedas de energia ou interrupções na fase de coleta de dados (COSTA, 2007).

Tais interferências produzem mudanças abruptas, que resultam na instabilidade dos dados e não são condizentes com o estudo das observações (YU et al., 2015). Todavia, a eliminação de dados deve ser feita cuidadosamente, pois pode resultar em perda de informações importantes para o desenvolvimento dos modelos.

Assim, utilizou-se o software Tsoft, para pré-processar os dados e eliminar alguns ruídos. Este software é dedicado à análise e processamento 
de séries temporais de gravidade e tem várias vantagens importantes na área de pré-tratamento dos dados (VAN CAMP \& VAUTERIN, 2005).

Com os dados filtrados, utilizou-se o software ETERNA34 para determinar a fase e a amplitude de cada componente harmônica. O pacote de processamento de dados Earth Tide ETERNA34 permite o registro, préprocessamento e análise de observações da Terra, a previsão de sinais de maré e o cálculo da carga de maré oceânica (WENZEL, 1996).

Aplicou-se então os valores obtidos das componentes harmônicas novamente no software TSoft para gerar o modelo sintético de maré terrestre e o modelo de carga oceânica e, após subtração destes dois modelos, removeuse o efeito da carga oceânica, gerando-se o novo modelo de maré terrestre.

As marés terrestres podem ser modeladas com precisão de poucos milímetros, utilizando-se de gravímetros absolutos e de medições GNSS, uma vez que os modelos preditos são extremamente precisos, por vezes superando a acurácia das medições de maré, porém estas medições de maré fornecem uma verificação desses modelos e, em alguns casos, permitem que eles sejam aprimorados (AGNEW, 2007). Isso significa que a maré terrestre pode ser calculada com precisão de cerca de 2 milímetros (HOOIJBERG, 2008). Coletou-se mais de 60.000 leituras gravimétricas em onze meses de observação, de março de 2013 a janeiro de 2014.

\subsection{Maré Oceânica}

O marégrafo foi instalado no ano de 1954 e desse programa permanente de medições, surgiram os primeiros trabalhos sobre o nível médio na região estuarina de Cananeia, e sobre as relações do nível do mar com os parâmetros meteorológicos em Cananeia (MESQUITA, 1997).

O registro das oscilações do nível do mar durante períodos longos, meses ou mesmo anos, permite materializar uma posição de equilíbrio como a média das observações. Se o nível do mar não se mantiver constante, este fato poderá ser constatado ao longo do tempo. O problema, entretanto, não é 
muito simples. É essencial controlar os processos dinâmicos da crosta nos locais onde os marégrafos estão instalados (BLITZKOW, 1998). Obteve-se as observações maregráfica de Cananeia (2012 a 2016) com o Instituto Oceanográfico da Universidade de São Paulo (IO-USP). Através das observações da altura de maré calculou-se o NMM no marégrafo, bem como sua tendência.

\subsection{Carga Oceânica}

Os movimentos das massas de água provocam alterações no campo gravitacional da Terra e a carga de água resulta em deslocamentos verticais adicionais. Ambos os fenômenos têm períodos de maré que não estão necessariamente em fase com as marés terrestres, porque a maré oceânica é restringida pela costa e pela topografia do fundo do oceano. Os harmônicos de maior amplitude são utilizados para calcular o efeito de carga oceânica na maré terrestre (AGNEW, 1997). O modelo de carga oceânica Finite Element Solution 95.2 (FES95.2) foi escolhido, pois este modelo é o que melhor se ajusta para o estado de São Paulo (COSTA, 2007).

Para obter-se este modelo, utilizou-se do sítio Ocean tide load provider do Observatório Espacial de Onsala (Bélgica), onde há a necessidade de se realizar algumas configurações, como: o tipo de formato de saída do arquivo texto, que neste caso foi o $B L Q$, além da unidade da gravidade ( $\mu \mathrm{m} / \mathrm{s} 2)(\mathrm{OSO}$, 2017). O modelo FES95.2, foi aplicado às coordenadas da estação geodésica de Cananeia.

Depois de receber o modelo de carga oceânica, fez-se necessário utilizar um conversor de $B L Q$ para o formato do Tsoft denominado "olgt.pl". Esse script computacional transforma o modelo em dados de amplitude e fase que podem ser inseridos no software Tsoft (OSO, 2017).

Outra correção necessária foi o movimento do polo, para tal fez-se o download dos dados de movimento do polo disponibilizados pelo International Earth Rotation and Reference Systems Service (IERS) (IERS,2013). O arquivo 
recebido pôde ser diretamente utilizado no software Tsoft, removendo assim a influência do movimento do polo nos modelos de maré terrestre.

\subsection{Nível Médio do Mar (NMM)}

O Nível Médio do Mar (NMM) é a altitude da superfície do mar medida em relação a um determinado ponto na superfície terrestre de referência. Esse mesmo NMM é de grande interesse para a Geodésia e áreas afins, onde o campo de gravidade da Terra determina o geoide, que é a superfície equipotencial no nível médio do mar que mais se aproxima do NMM no sentido do método dos mínimos quadrados. Se os oceanos estivessem em repouso (sem ondas, sem correntes, sem marés) o NMM coincidiria com o geoide (SNEEUW, 2006). Porém há uma diferença entre eles, chamada de Sea Suface Topography (SST), que não é abordada nesta pesquisa, para maiores detalhes ver Hayden et al. (2012). Apesar de parecer uma questão de simples resolução, a determinação da superfície de referência para algumas altitudes (ortométrica de Helmert, por exemplo) apresenta elevado grau de complexidade, pois o NMM sintetiza as influências de vários processos oceânicos: efeitos devido ao campo de massa, eventos meteorológicos, geopotencial terrestre e marés (MESQUITA, 2003).

Medições de nível do mar têm sido regularmente realizadas ao longo da costa brasileira e têm fornecido informações de grande relevância sobre variabilidades de longo período em dois aspectos principais: o nível médio do mar e as ondas de maré (MESQUITA, 2003). O nível médio do mar é calculado através de filtragens matemáticas das séries temporais, de modo a eliminar as oscilações de curto período, tais como as marés diurnas, semidiurnas, terdiurnas, etc. Interesse especial se encontra nas determinações dos níveis médios diários, mensais, sazonais e anuais, os quais, em geral, fornecem importantes indicações sobre variações hidrodinâmicas sazonais e tendências de longo período (HARARI et al., 2004). 
As séries temporais horárias de nível do mar em Cananeia foram editadas após plotagem e verificação computacional através da técnica do teste harmônico, desenvolvida por Karunaratne (1980) e Franco (1982). Os níveis médios diários, mensais, sazonais e anuais são calculados com a aplicação de um filtro passa baixa baseado em médias móveis: denotando A24 como o valor médio de 24 observações de modo a remover as oscilações de maré (GODIN,1972).

As leituras do nível do mar foram processadas e utilizaram-se destes filtros disponíveis no software PACMARÉ (desenvolvido por Franco) e disponibilizado pelo Instituto Oceanográfico da USP (HARARI et al., 2004).

O procedimento para o cálculo do NMM com o software PACMARÉ é simples: as observações maregráficas são tabuladas em um arquivo texto em uma única coluna (sequencialmente ano a ano), dento deste arquivo tem-se um cabeçalho constando o nome da estação maregráfica, latitude e longitude do marégrafo e a quantidade de observações contidas no arquivo. Este arquivo será utilizado no PACMARÉ na função "NMED”. O arquivo de saída contém os valores do NMM diário.

\subsection{Posicionamento por Ponto Preciso (PPP)}

O posicionamento por ponto preciso é o método de posicionamento GNSS em que se obtêm as coordenadas de um ponto utilizando apenas um receptor. O PPP elimina a necessidade de aquisição simultânea de dados de rastreamento a partir de uma estação de referência (base) e os problemas dependentes das distâncias que envolvem as linhas de base (NRCAN, 2007).

A ideia do método de PPP é a seguinte: órbitas precisas e relógios dos satélites são estimados, com base em observações de uma rede fiducial global de alta qualidade. Esta informação é tomada para resolver parâmetros da estação. Apenas uma única estação é processada de cada vez. Uma desvantagem é que o método é incapaz de ter em conta as correlações entre 
as estações e as órbitas (XU, 2007). As equações envolvidas no PPP de acordo com Lannes e Teunissen (2011) são: a equação de fase e da relação do código.

Os arquivos utilizados nesta pesquisa estão disponíveis gratuitamente no sitio do IBGE-RBMC (IBGE, 2016). Cada estação está denominada por um nome que indica o local onde esta se encontra. Assim, escolheu-se a estação NEIA para esta pesquisa, onde o local de estudo é Cananeia. Dessa forma, realizou-se o download dos arquivos dos anos de 2012 a 2016, que se encontram no banco de dados do IBGE e obteve-se uma série de 5 anos de dados GNSS da estação, onde tem-se um total de 1825 arquivos.

Para o processamento dos dados coletados da estação recorreu-se ao serviço de processamento on-line Canadian Spatial Reference System Precise Point Positioning (CSRS-PPP), disponibilizado pelo Canadian Geodetic Service of Natural Resources Canada (NRCAN), que fornece aos usuários a possibilidade de submeter através da internet, dados GNSS de simples ou dupla frequência em formato The Receiver Independent Exchange Format Version (RINEX), observados em modo estático ou cinemático. As coordenadas ( $\mathrm{E}, \mathrm{N}$ e h) e seus respectivos desvios padrão foram calculados e enviados para o e-mail do usuário. Para este trabalho utilizou-se do modo estático sem o modelo para correção de maré, e apenas as altitudes geométricas (h).

\section{Resultados e Discussão}

Conforme descrito na metodologia, cada um dos equipamentos utilizados para obtenção das observações, resultam em informações distintas (por exemplo, os dados da RBMC permitem obter a movimentação da crosta terrestre, enquanto que os dados de gravimetria nos permite calcular o modelo de maré terrestre). Porém, ao serem relacionadas verifica-se a interação entre elas. Dessa forma, para melhor entendimento será apresentado e discutido os resultados separadamente e depois seus relacionamentos para alcançar o objetivo do trabalho. 
O modelo teórico de maré terrestre de Wahr, Dehant e Defraigne (DEHANT et al., 1999) foi utilizado pelo software Tsoft para comparação com o modelo obtido de maré terrestre, porém o Tsoft não fornece os valores de amplitude para todas as componentes harmônicas contidas no modelo experimental. Para comparar as componentes do modelo teórico com as componentes do modelo experimental, foi necessário passar o modelo teórico pelo software ETERNA34 para obter-se as componentes que faltavam. A Tabela 1 mostra os valores para cada componente do modelo calculado. cujos significados dos campos da mesma são: amplitude teórica $\left(A_{t}\right)$ é obtida a partir de um modelo inelástico rígido para um planeta sem oceanos de WahrDehant; fator de amplitude $(F A)$; fase $(F)$ e seus respectivos desvios-padrão $\left(\sigma_{F A}\right.$ e $\left.\sigma_{F}\right)$. O fator de amplitude $(F A)$ multiplicado pela amplitude teórica $\left(A_{t}\right)$ determina a amplitude medida (A) (COSTA, 2007). Observam-se na Tabela 1 , os valores das principais componentes na geração do modelo de maré terrestre. As componentes O1, K1, M2 e S2 (destacadas em vermelho) foram as que melhor se ajustaram ao modelo. Elas também não obtiveram alterações significativas na fase, porém, apresentaram amplitudes ligeiramente maiores do que nos dados gravimétricos.

Para a componente harmônica lunar principal semi-diurna (M2), obteve-se uma amplitude de 71,70086 $\pm 0,00144 \mu \mathrm{Gal}$ e fase $2,3684 \pm 0,071^{\circ}$; e para a componente lunar principal diurna (O1), a amplitude obtida foi de $27,70202 \pm 0,00431 \mu \mathrm{Gal}$ e a fase $-1,5936 \pm 0,2122^{\circ}$. As componentes principais solar semi-diurna (S2) e lunar-solar diurna (K1) tiveram suas amplitudes e fases, respectivamente: $33,48414 \pm 0,00315 \mu \mathrm{Gal} ; 2,9008 \pm 0,1545^{\circ}$ e 37,89189 $\pm 0,00279 \mu \mathrm{Gal} ;-0,5527 \pm 0,1412^{\circ}$. A diferença entre o modelo teórico e o modelo experimental calculado, possui valor de $25 \mu \mathrm{Gal}(\sim 9 \mathrm{~cm})$ A Figura 2 mostra o comportamento da maré terrestre em Cananeia. 
Tabela 1- Amplitude e Fase das principais componentes do modelo experimental

\begin{tabular}{|c|c|c|c|c|c|c|c|c|}
\hline Onda & Freq. Min & Freq. Max & $A_{t}(\mu g a l)$ & $F A$ & $A(\mu g a l)$ & $\sigma_{F A}$ & $F\left({ }^{\circ}\right)$ & $\sigma_{F}$ \\
\hline $\mathrm{Q}_{1}$ & 0,50137 & 0,91139 & 4,55494 & 1,19964 & 5,464288 & 0,02043 & $-0,8223$ & 0,9756 \\
\hline $\mathrm{O}_{1}$ & 0,911391 & 0,947991 & 23,78999 & 1,16444 & 27,70202 & 0,00431 & $-1,5936$ & 0,2122 \\
\hline $\mathrm{M}_{1}$ & 0,947992 & 0,981854 & 1,87000 & 1,11484 & 2,084751 & 0,07048 & 1,7770 & 3,6227 \\
\hline $\mathrm{K}_{1}$ & 0,981855 & 1,023622 & 33,44386 & 1,133 & 37,89189 & 0,00279 & $-0,5527$ & 0,1412 \\
\hline $\mathrm{J}_{1}$ & 1,023623 & 1,057485 & 1,87069 & 1,17101 & 2,190597 & 0,05366 & 1,4051 & 2,6269 \\
\hline $\mathrm{OO}_{1}$ & 1,057486 & 1,470243 & 1,02330 & 1,31575 & 1,346407 & 0,15279 & 2,4033 & 6,6553 \\
\hline $2 \mathrm{~N}_{2}$ & 1,470244 & 1,880264 & 1,88513 & 1,17473 & 2,214519 & 0,03345 & 4,2871 & 1,6309 \\
\hline $\mathrm{N}_{2}$ & 1,880265 & 1,914128 & 11,80328 & 1,1837 & 13,97154 & 0,00708 & 1,8057 & 0,3428 \\
\hline $\mathrm{M}_{2}$ & 1,914129 & 1,950419 & 61,64687 & 1,16309 & 71,70086 & 0,00144 & 2,3684 & 0,071 \\
\hline $\mathrm{L}_{2}$ & 1,95042 & 1,984282 & 1,74263 & 1,17547 & 2,048409 & 0,05572 & 5,7897 & 2,7154 \\
\hline $\mathrm{S}_{2}$ & 1,984283 & 2,451943 & 28,67873 & 1,16756 & 33,48414 & 0,00315 & 2,9008 & 0,1545 \\
\hline $\mathrm{M}_{3} \mathrm{M}_{6}$ & 2,451944 & 7,000000 & 1,09707 & 1,27489 & 1,398644 & 0,02708 & $-2,8243$ & 1,217 \\
\hline
\end{tabular}

Figura 2 - Modelo de Maré Terrestre - Cananeia/SP

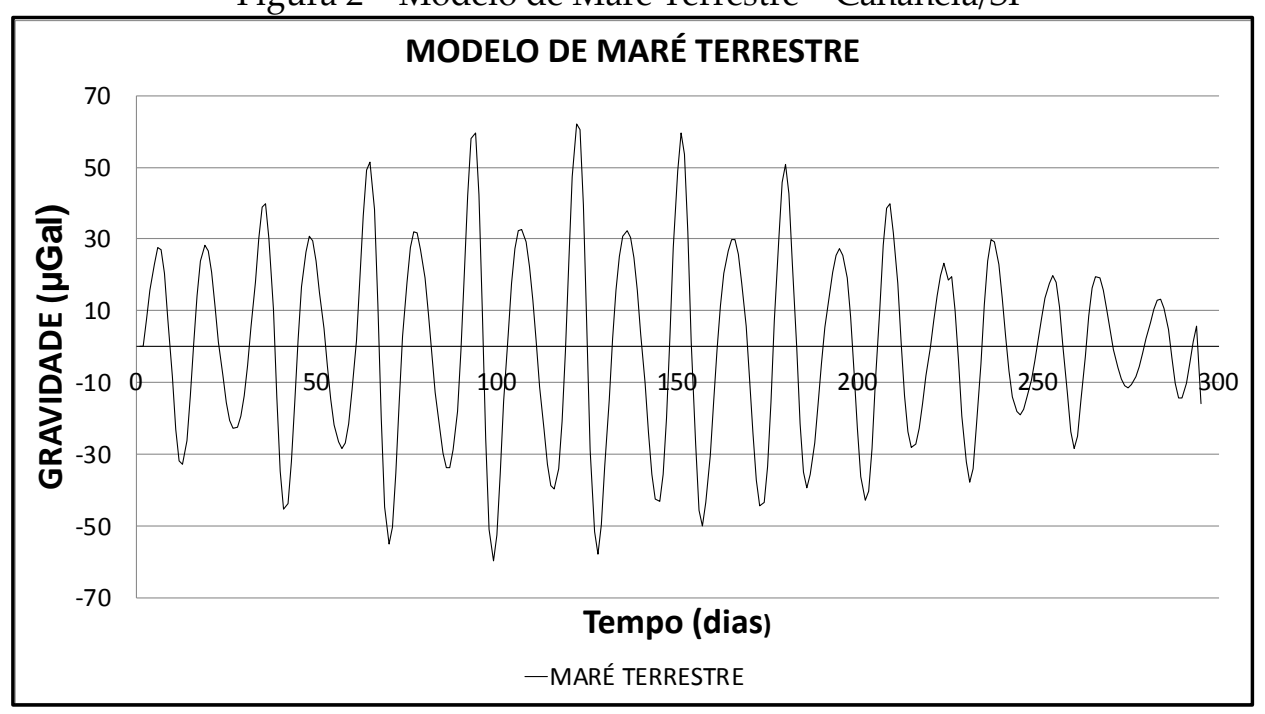

Fonte: elaborada pelos autores.

Realizou-se o cálculo e análise dos valores obtidos para a carga oceânica, utilizando-se o modelo FES95.2. Na Tabela 2 apresentam-se as amplitudes e fases deste modelo global de carga oceânica para a estação Cananeia.

Verifica-se que as principais componentes harmônicas (destacadas em vermelho) possuem os maiores valores, o que significa que a precisão do modelo está diretamente ligada à modelagem destas, além de também terem alterações significativas na fase. 
O modelo de carga oceânica, apresentado na Figura 3, mostra um intervalo de amplitude de aproximadamente $5 \mu \mathrm{Gal}$, o que é equivalente a uma variação de 1,6 cm de oscilação. A maré oceânica e terrestre tem origem no mesmo fenômeno gravitacional, porém com efeitos distintos, onde existe uma interação acentuada chamada de efeito indireto. Enquanto a maré terrestre apresenta uma variação gradual e bem modelada das componentes dos esforços em todo o corpo planetário sofrendo uma pequena influência das heterogeneidades mais superficiais, de forma diversa, a maré oceânica produz as sobrecargas superficiais e pouco efeito no interior planetário (CORDINI, 1998).

Essa afirmação pode ser verificada na Figura 4, onde observa-se a interação entre a carga oceânica e a maré terrestre, a porção terrestre tende a abaixar, o inverso também acontece quando esta sobrecarga deixa de agir e a superfície volta a seu estado de equilíbrio. Ao estudar as marés (terrestre e oceânica), observa-se que as marés terrestres também causam uma alteração na altura da maré oceânica devido a também agir no assoalho oceânico. Porém, o cálculo da Topografia da Superfície Oceânica (TSO), não é o objetivo deste trabalho, e será estudado e quantificado em trabalhos futuros.

Tabela 2 - Modelo de carga oceânica

\begin{tabular}{c|c|c|c}
\hline Onda & $\begin{array}{c}\text { Amplitude } \\
\left(\mathrm{m} / \mathrm{s}^{2}\right)\end{array}$ & $\begin{array}{c}\text { Amplitude } \\
(\mu \mathrm{gals})\end{array}$ & Fase $\left(^{\circ}\right)$ \\
\hline $\mathrm{M} 2$ & $3,09 \mathrm{E}-08$ & 3,090 & 67,65 \\
\hline $\mathrm{S} 2$ & $1,64 \mathrm{E}-08$ & 1,640 & 71,55 \\
\hline $\mathrm{K} 1$ & $3,03 \mathrm{E}-09$ & 0,303 & 93,52 \\
\hline $\mathrm{O} 1$ & $9,29 \mathrm{E}-09$ & 0,929 & 126,42 \\
\hline $\mathrm{N} 2$ & $7,04 \mathrm{E}-09$ & 0,704 & 55,65 \\
\hline $\mathrm{P} 1$ & $1,32 \mathrm{E}-09$ & 0,132 & 96,52 \\
\hline $\mathrm{K} 2$ & $3,78 \mathrm{E}-09$ & 0,378 & 80,65 \\
\hline $\mathrm{Q} 1$ & $2,45 \mathrm{E}-09$ & 0,245 & 144,72 \\
\hline $\mathrm{Mf}$ & $4,70 \mathrm{E}-10$ & 0,047 & $-143,80$ \\
\hline $\mathrm{Mm}$ & $3,40 \mathrm{E}-10$ & 0,034 & $-169,60$ \\
\hline Ssa & $1,90 \mathrm{E}-10$ & 0,019 & $-178,40$ \\
\hline \multicolumn{4}{c}{ Fonte: elaborada pelos autores. }
\end{tabular}


Figura 3 - Modelo de Carga Oceânica - Cananeia/SP

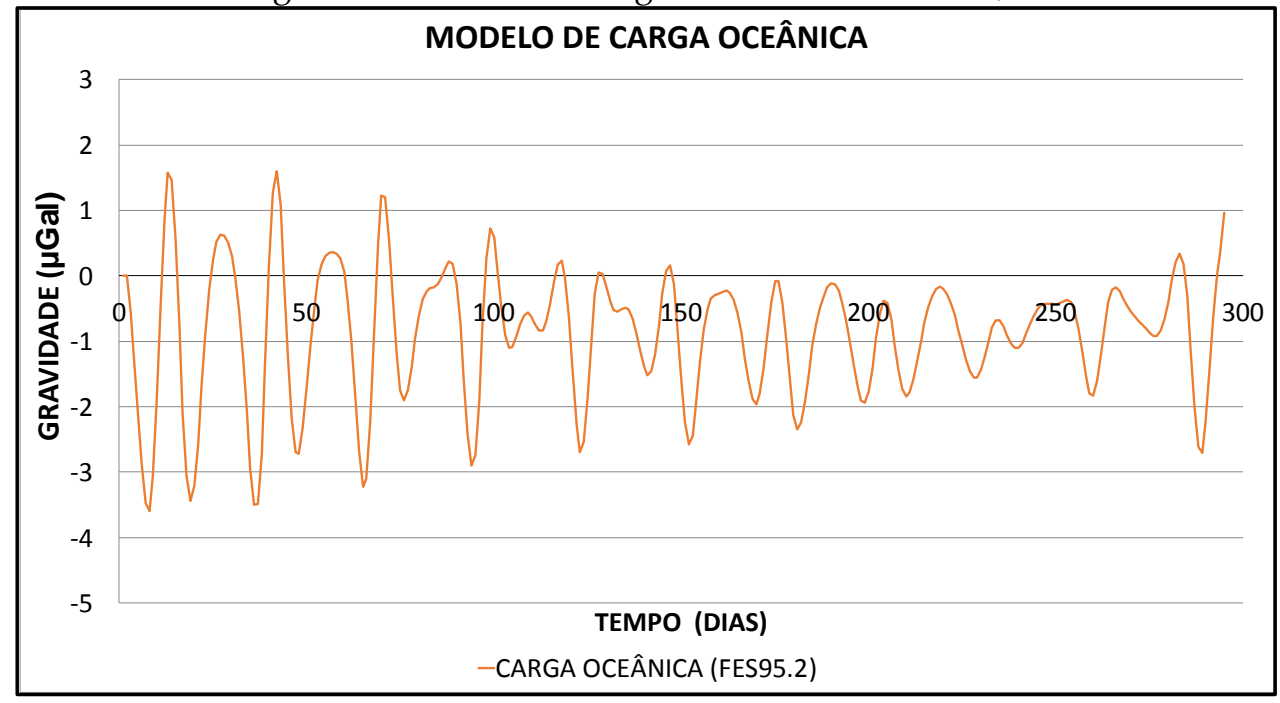

Fonte: Autor (2017).

Figura 4 - Interação da Carga Oceânica com a Maré Terrestre

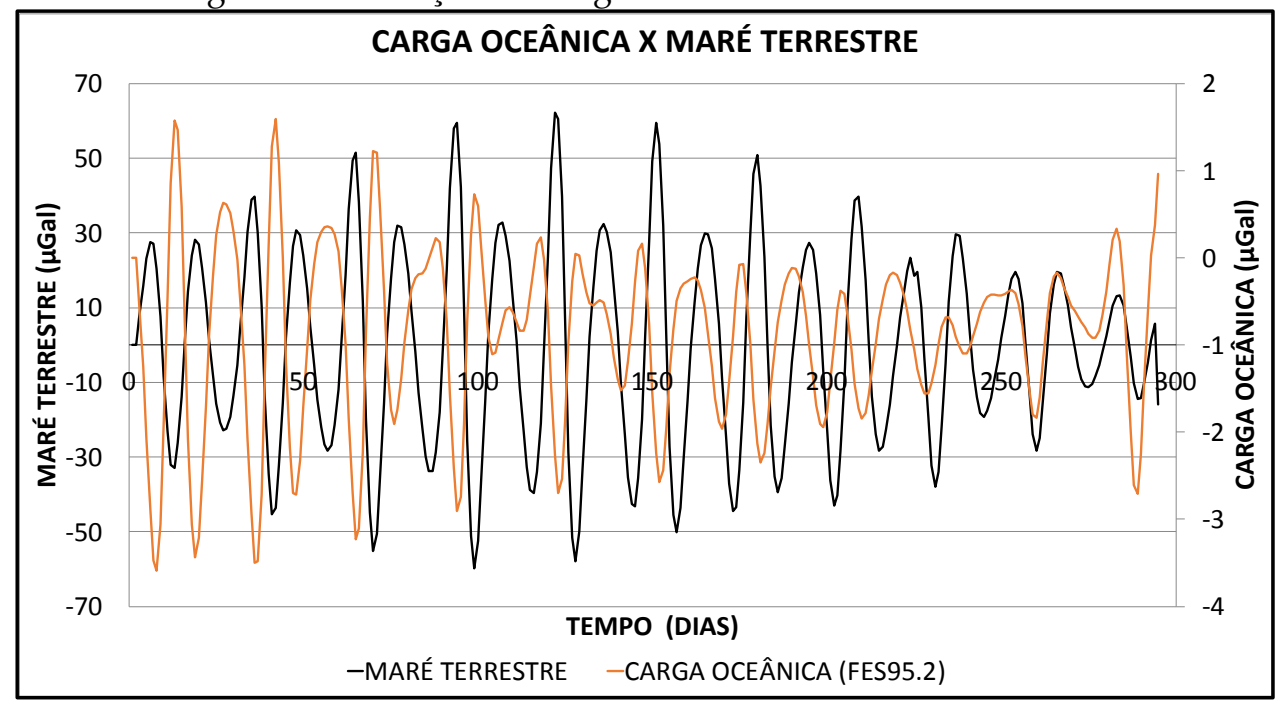

Fonte: elaborada pelos autores.

Calculou-se o nível médio do mar para Cananeia, através das observações maregráficas onde verifica-se que o nível médio do mar possui valor de 249,11 cm com tendência de aumento constante, com variação de $2 \mathrm{~mm}$ /ano. Esta variação é a mesma quando comparada com trabalhos anteriores de Trabanco et al. (2003), Trabanco et al. (2005), Blitzkow (1998) e Blitzkow et al. (2007). A Figura 5 mostra os resultados obtidos para o NMM diário, onde se percebe que a tendência vem aumentando gradativamente. 
Figura 5 - Comportamento do Nível Médio do Mar (NMM)

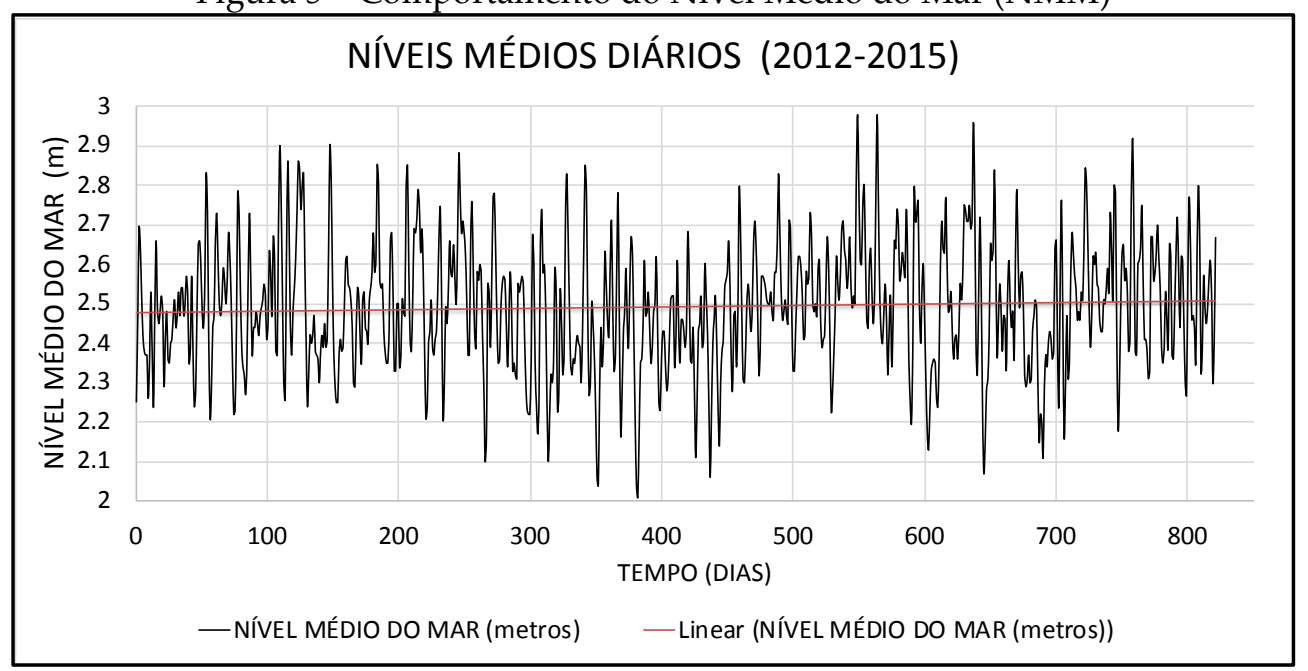

Fonte: elaborada pelos autores.

Realizou-se a análise da estabilidade da estação RBMC - NEIA, através do processamento das observações GNSS, onde foi extraída a altitude geométrica, assim pode-se quantificar e verificar com precisão a existência ou não da movimentação da crosta. A Figura 6 mostra os resultados obtidos do processamento GNSS pelo método PPP, ao se analisar a variação da altitude geométrica, constata-se que há movimentação da crosta.

Apesar dos valores máximo e mínimos da Figura 6 serem respectivamente $+3,0 \mathrm{~cm}$ e $-0,7 \mathrm{~cm}$, observa-se que a linha de tendência possui valor, da movimentação da crosta, de $2 \mathrm{~mm}$ para esta série de 5 anos (20122016). Os valores apresentados reforçam os estudos anteriores sobre a movimentação e estabilidade da estação NEIA, e as referências bibliográficas são comprovadas mostrando a potencialidade do posicionamento por ponto preciso na geodinâmica.

Ao se analisar os valores obtidos entre o NMM e o PPP, pode-se verificar que os eles possuem o mesmo valor de tendência (2 $\mathrm{mm})$, isso implica em dizer que essa confrontação mostra a solidez da estação NEIA, e que a mesma tem uma variação constante de aumento ao ano. 
Figura 6 - Comportamento da altitude geométrica da Estação RBMC-NEIA

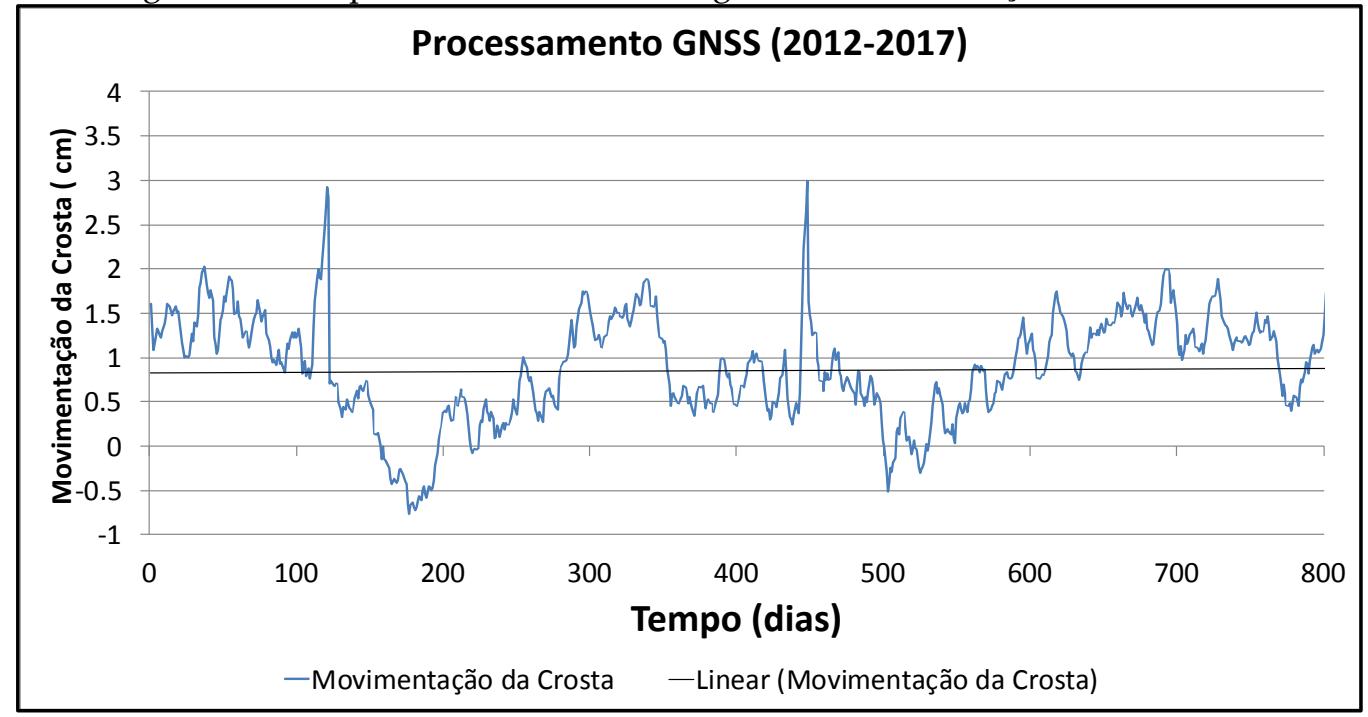

Fonte: elaborada pelos autores.

As altitudes obtidas através do PPP têm alta acurácia, pois as mesmas podem ser confirmadas pela solução que o IBGE produz. Esta solução é realizada semanalmente em dezenas de estações que compõem a rede Sistema de Referência Geocêntrico para as Américas (SIRGAS2000). Os dados são processados no modo relativo utilizando estações International GNSS Service (IGS) e o software científico Bernese 5.0 (IBGE, 2016). Uma comparação com o processamento do IBGE foi realizada com objetivo de avaliar o método de posicionamento utilizado (PPP), verificou-se que o comportamento da crosta é o mesmo da solução produzida pelo IBGE.

\section{Conclusão}

Após os vários resultados multiparamétricos obtidos e descritos ao longo deste trabalho, cabe enfatizar que todas as fontes de observações utilizadas, mesmo que em algum momento pareçam divergir, os resultados visaram propiciar as condições para evidenciar os efeitos geodinâmicos aos quais a estação RBMC-NEIA e o marégrafo estão sujeitas.

As análises sobre a deformação da crosta terrestre (Figura 6) indicam uma movimentação vertical, sendo que esta movimentação (conforme já 
apresentado) possui valor estável, ou seja, tem uma variação pequena ao longo da série analisada. Se olharmos para valores absolutos dos valores máximo e mínimo, temos uma variação da altitude geométrica de $\sim$ 3,7 centímetros.

A carga oceânica está diretamente ligada com a deformação da crosta e maré terrestre, havendo a necessidade de ser bem modelada (Figura 3) para que sejam realizadas as correções na altitude das estações RBMC, tendo seu valor de 1,6 centímetros, o que à torna a estação NEIA com pouca movimentação, quando confrontada com os resultados do PPP.

As estações geodésicas são utilizadas como rede primária para trabalhos de infraestrutura, e estão à mercê de todas as influências geodinâmicas presentes no ambiente onde se localizam, por isso ao se trabalhar com projetos que demandam alta precisão é necessário modelar esses erros ou efeitos geodinâmicos para viabilizar a execução de grandes obras. Já a maré terrestre tem uma boa modelagem em todo o globo terrestre, variando pouco, porém há necessidade de realizar sua modelagem geodinâmica, principalmente em áreas costeiras. No cálculo da maré terrestre (Figura 2), verifica-se que esta teve sua modelagem com alta acurácia, onde se pode verificar que os comportamentos das principais componentes (M2 e O1) na geração do modelo experimental de maré terrestre se ajustaram ao modelo teórico.

Segundo Freitas e Cordini (1998), em algumas regiões do globo, a mensuração do efeito de resposta regional às marés terrestres, quando comparados aos efeitos de maré modelados (maré teórica), pode apresentar diferenças de até $30 \mu \mathrm{Gal}$ ou $10 \mathrm{~cm}$, levando-se em consideração que a precisão das medidas de gravidade, nos dias atuais, pode atingir $1 \mu \mathrm{Gal}$. Dessa forma, o modelo de maré terrestre obtido neste trabalho atende o nível de precisão deste tipo de mensuração nesta região, ficando com diferenças de $25 \mu \mathrm{Gal}$, objetivando a determinação da resposta crustal verdadeira, o que proporcionalmente gera uma diferença de $\approx 9,0 \mathrm{~cm}$. Esta variação pode ser vista na figura 4, onde a interação entre a carga oceânica e a maré terrestre 
tem efeito contrário, fazendo a crosta terrestre ter movimentação ao longo do dia. Para trabalhos futuros, um período maior de observações gravimétricas deve ser realizado, permitindo uma melhor determinação das componentes de longo período, o que produzirá modelos mais precisos.

$O$ valor calculado para o NMM é de 2,491 metros, e sua tendência é um aumento de $2 \mathrm{~mm} / \mathrm{ano}$, o que mostra que as observações maregráficas possui boa acurácia e proporcionam determinar esta pequena variação ao longo da série utilizada (apenas 5 anos). Normalmente, para uma boa acurácia do NMM uma série longa (de 18,6 anos) e sem interrupções nas observações é a mais indicada, pois cada componente harmônica de maré possui um período específico, onde a mais longa é chamada de período nodal da Lua. Em termos absolutos os valores máximo e mínimo do NMM são, respectivamente, de 2,95 m e 2,00 m, o que dá uma amplitude de 95 centímetros para a estação Cananeia (Figura 5).

Conforme os resultados obtidos para cada uma das fontes (marés, PPP, NMM) pode-se dizer que todas são potenciais fontes de erros e que tem forte interação entre si. A não modelagem geodinâmica ou a negligência destes efeitos podem resultar em diferenças significativas, por exemplo, na altitude geométrica da estação da RBMC, uma vez que a estação está sobre influência direta destas cargas.

Determinaram-se os modelos e calcularam-se os valores das influências geodinâmicas na movimentação da crosta através das observações GNSS, maré terrestre e oceânica, efeito da carga oceânica e realizou-se o cálculo do nível médio do mar para Cananeia, e verificou-se que há uma forte interação entre estas fontes de observações, onde as análises dos valores obtidos nas várias fontes comprovam sua interação e os resultados apontam para a estabilidade da estação e aumento do nível médio do mar.

Portanto, os objetivos propostos foram alcançados através dos resultados apresentados e os modelos obtidos condizem com a realidade geodinâmica da estação e o NMM tem pouca variação, onde mantém um 
aumento contínuo ao longo do tempo e as estações GNSS e maregráfica possuem confiabilidade e estabilidade em suas observações.

\section{Agradecimentos}

Os autores agradecem à FEC/Unicamp, à UFU - Campus Monte Carmelo e à Geodesy and Geomatic Engineering - University of New Brunswick pelo apoio durante o período do Sandwich Course. E ao IFSULDEMINAS pela licença do primeiro autor para desenvolver o doutorado. O presente trabalho foi realizado com apoio da Coordenação de Aperfeiçoamento de Pessoal de Nível Superior - Brasil (CAPES) - Código de Financiamento 001 (processo PDSE nº 88881.132305/2016-01).

\section{Referências}

AGNEW, D. C. Earth Tides. University of California San Diego, CA, USA. Elsevier B.V. p $163-191,2007$.

BAKER, T. F.; CURTIS, D. J.; DODSON, A. H. A new test of Earth tide models in central Europe. Geophysical research letters.1996.

BLITZKOW D. Monitoramento das Condições Geodinâmicas dos Marégrafos de Cananeia e Ubatuba: Brasil, Lat (24 S). www.mares.io.usp.br/aagn/- ISSN 1983-0319. Afro-América GLOSS News, 3. 1998.

BLITZKOW, D. CAMPOS. I. O. DE FREITAS. S. R. C. Altitude: O que interessa e como equacionar?. Departamento de Engenharia de Transportes - Escola Politécnica, Universidade de São Paulo, São Paulo. 2007.

BRETREGER, K. MATHER. R. S. Modelling Ocean-loading Effects on Tidal Gravity in Australia. Geophysical Journal of the Royal Astronomical Society, DOI: 10.1111/j.1365-246X.1978.tb04232.x. 52: p. 241-257. 1978.

CORDINI, J. Estudos dos Aspectos Geodinâmicos no Datum Vertical do SGB. Curitiba, 159 p. Tese (Doutorado em Ciências Geodésicas) - Departamento de Geomática, Universidade Federal do Paraná. 1998. 
COSTA, D. S. Variação do Nível Médio do Mar - Técnicas para a Avaliação.

Dissertação de Mestrado em Engenharia de Transportes - Escola Politécnica, Universidade de São Paulo, São Paulo. 2007.

DEHANT, V. DEFRAIGNE, P. WAHR, J. M. Tides for a convective Earth. Journal of Geophysical Research: Solid Earth, v. 104, n. B1, p. 1035-1058. 1999.

FRANCO, A. S. "Au sujet de la méthode de vérification des hauteurs horaires de marée de Karunaratne" - Révue Hydrographique Internationale, Mônaco, LIX (1), p. 151-155. 1982.

FREITAS S. R. C. CORDINI J. Implicações Geodinâmicas no Posicionamento Geodésico. Boletim de Ciências Geodésicas. 1998.

GEMAEL, C. Introdução à Geodésica Física. $2^{\mathrm{a} e d}$. Curitiba, PR: Editora da UFPR. ISBN 85-7335-029-6. 2002.

GODIN, G. The analysis of tides - Liverpool University Press, 246 p. 1972.

HARARI, J. FRANÇA, C.A.S. CAMARGO, R. Variabilidade de longo termo de componentes de marés e do nível médio do mar na costa brasileira. AfroAmerica Gloss News. 2004.

HAYDEN T., RANGELOVA, E., SIDERIS, M.G., VÉRONNEAU, M. Contribution of Tide Gauges for the Determination of $\mathrm{W}_{0}$ in Canada. Gravity, Geoid and Height Systems, Proceedings of the IAG Symposium GGHS2012, Venice, Italy. p. 241-256, 2012.

HOOIJBERG, M. Information and Computer Technology. Geometrical Geodesy:

Using Information and Computer Technology, p. 289-295. 2008.

IBGE, Rede Brasileira de Monitoramento Contínuo dos Sistemas GNSS. 2016.

Disponível em:

<http://www.ibge.gov.br/home/geociencias/geodesia/rbmc/rbmc.shtm> Acesso: 26 setembro 2016.

INTERNATION EARTH ROTATION AND REFERENCE SYSTEMS SERVICE (IERS). 2013. Earth orientation data. Disponível em:<http://www.iers.org/IERS/EN/DataProducts/EarthOrientationData/eop.h tml.> Acesso em 18 Janeiro 2017.

KARUNARATNE, D. A. An improved method for smoothing and interpolating hourly sea level data" - International Hydrographic Review, vol 57 (1), p. 145-158. 1980. 
LANNES, A.; TEUNISSEN, P. J. G. GNSS algebraic structures. Journal Geodesy, [s.l.], v. 85, n. 5, p.273-290, Springer Science + Business Media. 2011.

LAZZARO, D. Ressonâncias Planetárias. Rio de Janeiro: Publicação do Observatório Nacional. 1989.

MELCHIOR, P. The Tides of the Planet Earth. [S.l]: Pergamon Press. 1983.

MESQUITA, A.R. Marés, Circulação e Nível do Mar na Costa Sudeste do Brasil,

IOUSP, Documento Preparado para A FUNDESPA (Fundação de Estudos e Pesquisas $\quad$ Aquáticas). $1997 . \quad$ Disponível em <http://www.mares.io.usp.br/sudeste/sudeste.html> Acesso em 05 março 2016. MESQUITA, A. R. Sea level variations along the Brazilian coast: a short review Journal of Coastal Research, SI 35, p. 21-31. 2003.

NATURAL RESOURCE CANADA (NRCAN). 2007. CSRS-PPP an online Global GPS Processing Service. Canadian Spatial Reference System, Geodetic Survey Canada. Disponível em: <https://www.unavco.org/projects/projectsupport/polar/.../PPP.pdf> Acesso:19 maio 2016.

ONSALA SPACE OBSERVATORY (OSO). 2017. Welcome to the free ocean tide loading provider. Disponível em: < http://holt.oso.chalmers.se/loading/> 16 jan 2017.

PINTO, J. V. Comparison of Models for the Calculation of Orbital Perturbation due to the Terrestrial Tide. Dissertação de Mestrado em Física - Faculdade de Engenharia do Campus de Guaratinguetá, Universidade Estadual Paulista, Guaratinguetá. 2005.

RAMOS. M.P; CARMO, E.J.; DAL POZ, W.R.; CAMARGO. P.O. Análise do efeito de carga oceânica no posicionamento por ponto preciso. VIII Colóquio Brasileiro de Ciências Geodésicas, Curitiba. 2013.

SEEBER, G. Satellite Geodesy: foundations, methods, and applications. 2nd. ed.,Berlin,New- York:Walter de Gruyter. 2003.

SNEEUW, N., Physical Geodesy, Institute of Geodesy - Universitat Stuttgart, 2006.

TRABANCO, J. L. A. FREITAS, S. R. C. BLIZKOW, D. SCHUAB, S. Periodical local load effects and geocentric positioning of Cananeia tide gauge, Brazil. In: IUGG - XXIII General Assembly of Geodesy and Geophysical Society, Sapporo - Japan. 2003. 
TRABANCO, J. L. A. BLITZKOW, D. FRANÇA, C.A. S. MESQUiTA, R. A de. Analysis of the sea level from a 50 years tide gauge Record and GPS. In: Dynamic Planet 2005 IAG/IAPSO/Iabo Joint Assembly, 2005, Caims, Austrália. Dynamic Planet, IAG/IAPSO/Iabo Joint Assembly. 2005.

VAN CAMP, M; VAUTERIN, P. Tsoft: graphical and interactive software for the analysis of time series and Earth tides, Computers \& Geosciences, pag. 631640. 2005.

YU, H. J., GUO, J. Y., LI, J. L., MU, D. P., KONG, Q. L. Zero Drift and Solid Earth Tide extracted from Relative Gravimetric data with Principal Component Analysis. Geodesy and Geodynamics, vol. 6, n², p 143 -150. 2015.

WENZEL, H G. The nanogal software: Earth tide data processing package ETERNA 3.3. (Bull. d'Inf. Marées Terr) 9425-9439. 1996. Disponivel em: <http://www.eas.slu.edu/ggp/eterna34/manual/eterna33.htm> Acesso em 18 de Janeiro de 2017.

XU, G. Orbits. Germany: Springer-Verlag Berlin Heidelberg, . ISBN: 978-3-54078521-7. 2007. 